MITSUBISHI ELECTRIC RESEARCH LABORATORIES

http://www.merl.com

\title{
Multi-Pilot Aided Carrier Phase Estimation for Single Carrier Coherent Systems
}

\author{
Pajovic, M.; Millar, D.S.; Koike-Akino, T.; Kojima, K.; Arlunno, V.; Parsons, K.
}

TR2015-080 June 26, 2015

\begin{abstract}
We propose a novel low complexity CPE algorithm that uses pilot symbols and inference for estimating carrier phase in single carrier systems. Performance is validated by simulation of 64QAM over the range of SNRs for which 64QAM is optimal for BICM.
\end{abstract}

2015 Signal Processing in Photonic Communications (SPPCom)

This work may not be copied or reproduced in whole or in part for any commercial purpose. Permission to copy in whole or in part without payment of fee is granted for nonprofit educational and research purposes provided that all such whole or partial copies include the following: a notice that such copying is by permission of Mitsubishi Electric Research Laboratories, Inc.; an acknowledgment of the authors and individual contributions to the work; and all applicable portions of the copyright notice. Copying, reproduction, or republishing for any other purpose shall require a license with payment of fee to Mitsubishi Electric Research Laboratories, Inc. All rights reserved. 



\title{
Multi-Pilot Aided Carrier Phase Estimation for Single Carrier Coherent Systems
}

\author{
Milutin Pajovic, David S. Millar, Toshiaki Koike-Akino, \\ Keisuke Kojima, Valeria Arlunno, Kieran Parsons \\ Mitsubishi Electric Research Laboratories (MERL), 201 Broadway, Cambridge, MA, 02139, USA. \\ pajovic@merl.com
}

\begin{abstract}
We propose a novel low complexity CPE algorithm that uses pilot symbols and inference for estimating carrier phase in single carrier systems. Performance is validated by simulation of 64QAM over the range of SNRs for which 64QAM is optimal for BICM.
\end{abstract}

OCIS codes: 060.4510 Optical communications, 060.1660 Coherent communications, 000.5490 Probability theory, stochastic processes, and statistics

\section{Introduction}

The recent development of second and third generation coherent optical transmission systems has been driven by a desire to increase both noise tolerance and spectral efficiency. While first generation coherent systems relied on dualpolarization quaternary phase shift keying (DP-QPSK) [1], which has high phase noise tolerance, this limits spectral efficiency to $4 \mathrm{~b} / \mathrm{s} / \mathrm{Hz}$, less overheads for coding and framing. This has led to a wide scale adoption of quadrature amplitude modulation (QAM), with multiple levels of signal per quadrature. Cutting-edge experiments are now using DP- 16-, 64-, and 256-QAM modulation. With the increased density of modulation, carrier phase estimation (CPE) has become an increasing problem. These dense modulation formats are sensitive to phase noise, and yet they are unsuited to blind algorithms such as the Viterbi and Viterbi algorithm [3] which was so successful with DP-QPSK systems [2]. This has led to a wide variety in proposed CPE algorithms [4,5], many of which are decision-directed (i.e. phase due to modulation is removed with one or more symbol decisions in the CPE algorithm). One approach that has generated significant interest in the research community is that of two-stage phase estimation [6,7]: first, a coarse estimation is made with one algorithm, unwrapped and applied; then a more accurate algorithm is applied to provide a final estimate without the need for symbol-by-symbol unwrapping. Another approach recently developed is using the phase noise statistics inferred from one or more pilot symbols to calculate log-likelihood ratios more accurately for orthogonal frequency division multiplexing (OFDM) signals [8].

In this paper, we propose a two-stage pilot-aided scheme for CPE, suitable for operation in the low SNR regime which is optimal for bit-interleaved coded modulation (BICM). The initial estimate of phase is inferred on a block basis from multiple pilots using Kalman smoothing [9]. This initial estimate is then refined by performing expectationmaximization in parallel for the information symbols in the block. Finally, an averaging filter is used on the phase estimate, before the estimated phase is applied to the signal. These algorithms are of moderate complexity, and are suitable for block-based parallel implementation in hardware. The performance of our proposed algorithm is validated by simulation over the SNR region for which 64-QAM is optimal for bit-interleaved coded modulation.

\section{Proposed Algorithm}

\subsection{Channel and Phase Noise Model}

Assuming all signal impairments but phase noise have been compensated, a sample of the received signal at discrete time $n, y_{n}$, is modeled as

$$
y_{n}=x_{n} e^{j \theta_{n}}+v_{n}, \quad \text { where } v_{n} \sim \mathscr{C} \mathscr{N}\left(0, \sigma_{v}^{2}\right), \quad \theta_{n}-\theta_{n-1} \sim \mathscr{N}\left(0, \sigma_{\mathrm{p}}^{2}\right), \quad \text { and } \sigma_{\mathrm{p}}^{2}=2 \pi \Delta v T_{\mathrm{s}},
$$

where $x_{n}$ is the transmitted symbol chosen uniformly from some constellation $\mathscr{X}, \Delta v$ is the combined linewidths of transmitter's laser and local oscillator, and $T_{\mathrm{S}}$ is the duration of the signaling interval. 


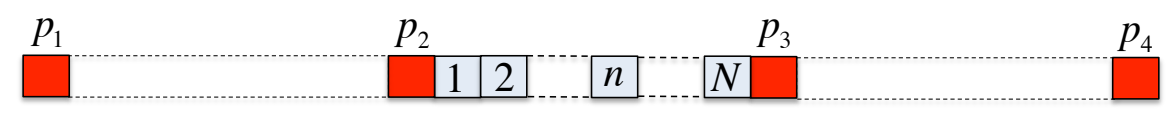

Fig. 1. Illustration of multi-pilot estimation with $2 K=4$ pilots.

\subsection{Processing Stage 1: Inference of Pilot Symbol Phases}

We assume that $N$ information symbols are transmitted in a block and each block starts with a pilot symbol. To estimate the phase of a symbol transmitted during the $n$-th signaling interval, $K$ pilots preceding and $K$ pilots following the considered symbol are used. Figure 1 depicts an example where $K=2$ and phase estimation of information symbols between pilots $p_{2}$ and $p_{3}$ is performed with the aid of pilots $p_{1}, p_{2}, p_{3}$ and $p_{4}$.

The pilot processing stage starts with approximating the posterior distribution $p\left(\theta_{p_{k}} \mid x_{p_{k}}, y_{p_{k}}\right)$ of pilot $p_{k}$ 's phase, conditioned on the transmitted symbol $x_{p_{k}}$ and received signal $y_{p_{k}}$. Since direct application of Bayes' rule on model (1) does not admit a closed form expression, we approximate the posterior with Gaussian distribution such that $p\left(\theta_{p_{k}} \mid x_{p_{k}}, y_{p_{k}}\right) \sim \mathscr{N}\left(\mu_{p_{k}}, \sigma_{p_{k}}^{2}\right)$, where mean $\mu_{p_{k}}$ is the Maximum Likelihood phase estimate, while the variance $\sigma_{p_{k}}^{2}$ is evaluated as an observed Fisher information [9].

The distributions of pilot phases are then smoothed by accounting for phase noise statistics. This is achieved by processing the posteriors $p\left(\theta_{p_{k}} \mid x_{p_{k}}, y_{p_{k}}\right), k=1, \ldots, 2 K$, through Kalman filter via full forward pass from pilot $p_{1}$ to $p_{2 K}$, and backward pass from pilot $p_{2 K}$ to $p_{K+1}$. On an implementation side, Kalman filter used here can either be realized as a sequential processor over $2 K$ scalars, or as a parallel processor which performs inversion of a matrix of size $2 K$ on each pilot. Our study shows that increasing the number of pilots $2 K$ above some relatively small value, which could be as low as 4 in most scenarios with $\Delta v T_{\mathrm{s}}$ 's encountered in practice, yields negligible performance gains. Therefore, either implementation of Kalman filtering does not lead to excessive delays or unreasonable increase in required computations.

\subsection{Processing Stage 2: Estimation of Information Symbol Phases}

The second processing stage starts with inferring phases of information symbols using the Gaussian phase posteriors corresponding to pilots $p_{K}$ and $p_{K+1}$, obtained in the previous stage, and phase noise statistics. Omitting the details, it is shown that the information symbol $n$ 's phase, $\theta_{n}$, can be summarized as $p\left(\theta_{n} \mid x_{p_{1}}, y_{p_{1}}, \ldots, x_{p_{2 K}}, y_{p_{2 K}}\right) \sim \mathscr{N}\left(\mu_{n}, \sigma_{n}^{2}\right)$, where $\mu_{n}$ depends on the means and variances of Gaussian posteriors corresponding to pilots $p_{K}$ and $p_{K+1}$, obtained at the output of Kalman filter.

The initial estimates $\mu_{n}$ of information symbol phases are refined by employing the Expectation Maximization (EM) procedure on each symbol separately in parallel. The EM routine corresponding to the $n$-th information symbol is initialized with $\hat{\theta}_{n}^{(0)}=\mu_{n}$. The $k$-th iteration evaluates the likelihood of symbol $x_{n}$ given the received signal $y_{n}$ and phase estimate $\theta_{n}^{(k-1)}$ from the previous iteration. Then, the symbol likelihoods are used to update the corresponding phase estimates. The EM procedure is terminated after some predefined number of iterations. Our study shows that the EM algorithm converges fairly quickly so that after already 2 iterations the performance gains become negligible.

In the final processing step, the EM phase estimates are filtered using a moving average filter, which yields the final phase estimates of information symbols. The soft and hard decisions of the transmitted symbols then directly follow from model (1) and final phase estimates.

\section{Simulation Results}

In order to determine the appropriate SNR regime for which 64-QAM is optimal, we simulated the Generalized Mutual Information (GMI) [10] for 16-QAM, 64-QAM and 256-QAM over an AWGN channel. The results of this simulation are presented in Fig. 2(a). We noted that 64-QAM is optimal between approximately $10 \mathrm{~dB}$ and $17 \mathrm{~dB}$ SNR. This corresponds to the range of SNRs for which 64-QAM has the best spectral efficiency of the square-QAM formats, assuming the use of BICM.

We then simulated the performance of 64-QAM, with varying $\Delta v T_{s}$ from $10^{-5}$ to $10^{-4}$. We compared the performance of the D-PLL algorithm presented in [4], with the algorithm proposed in this paper. The results of these simulations are presented in Fig. 2(b) and 2(c) respectively. We note that while the D-PLL algorithm has satisfactory performance at low $\Delta v T_{s}$, it fails rapidly as $\Delta v T_{s}$ is increased beyond $2 \times 10^{-5}$. By comparison, our algorithm is remarkably robust in the presence of high levels off additive noise and phase noise. We note that for $\Delta v T_{s}=10^{-4}$, we 


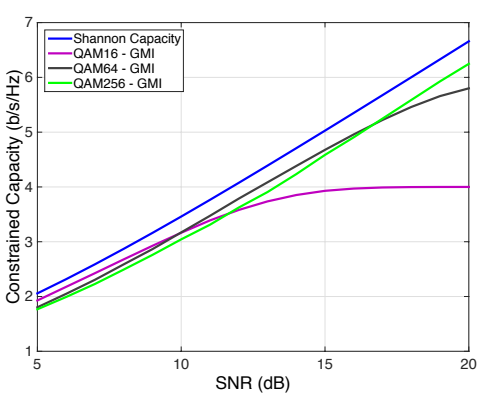

(a)

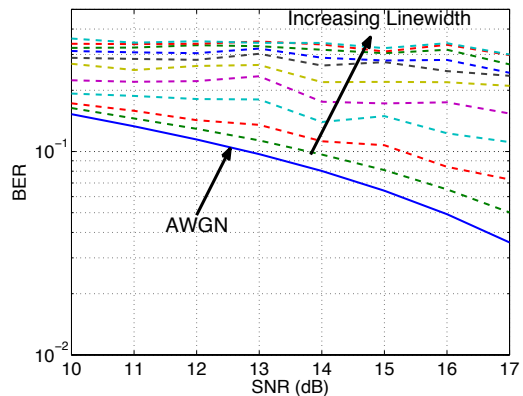

(b)

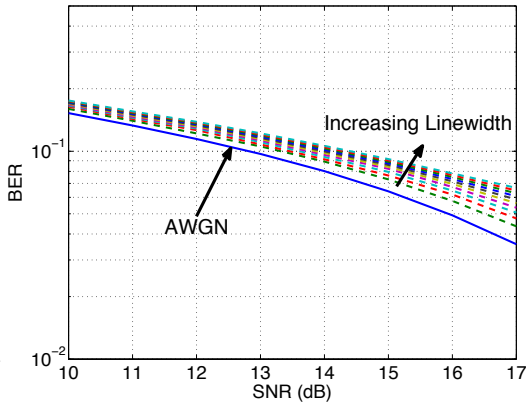

(c)

Fig. 2. (a) Capacity of different square QAM modulation formats with AWGN channel, assuming BICM. Performance of 64QAM over the BICM optimal region with AWGN only, and $\Delta v T_{s}$ from $10^{-5}$ to $10^{-4}$ is plotted for (b) D-PLL carrier recovery, and (c) our proposed algorithm.

see a penalty of less than $2 \mathrm{~dB}$ at a BER of 0.1 . This corresponds to an SNR improvement of more than $3 \mathrm{~dB}$ over the D-PLL algorithm.

\section{Conclusions}

We have proposed a pilot aided carrier phase recovery algorithm for single carrier, coherent systems. The algorithm utilizes multiple pilots for estimating phase rotation of an information symbol, relies on statistical inference techniques and admits parallel implementation in hardware. We determined the optimal SNR region for BICM performance of 64-QAM, and demonstrated significant performance improvements compared with the D-PLL algorithm.

\section{References}

1. C. R. S. Fludger et al., "Coherent Equalization and POLMUX-RZ-DQPSK for Robust 100-GE Transmission," J. Lightw. Tech., 26, 2008, pp 64-72.

2. R. Noé, "Phase Noise-Tolerant Synchronous QPSK/BPSK Baseband-Type Intradyne Receiver Concept With Feedforward Carrier Recovery," J. Lightw. Tech., 23, 2005, pp 802-808.

3. A. J. Viterbi and A. M. Viterbi, "Nonlinear estimation of PSK-modulated carrier phase with application to burst digital transmission," IEEE Trans. Inf. Th., 29, 1983, pp 543-551.

4. I. Fatadin et al., "Blind Equalization and Carrier Phase Recovery in a 16-QAM Optical Coherent System," J. Lightw. Tech., 27, 2009, pp 3042-3049.

5. T. Pfau et al., "Hardware-Efficient Coherent Digital Receiver Concept With Feedforward Carrier Recovery for M-QAM Constellations," J. Lightw. Tech., 27, 2009, pp 989-998.

6. S. M. Bilal et al., "Dual Stage CPE for 64-QAM Optical Systems Based on a Modified QPSK-Partitioning Algorithm," Photon. Tech. Lett., 2014, pp 267-270.

7. T. Pfau and R. Noé, "Phase-Noise-Tolerant Two-Stage Carrier Recovery Concept for Higher Order QAM Formats," IEEE JSTQE, 16, 2010, pp 1210-1216.

8. S. Cao et al., "Pilot-Tone Assisted Log-Likelihood Ratio for LDPC Coded CO-OFDM System," IEEE PTL, 26, 2014, pp 1577-1580.

9. C. M. Bishop, "Pattern Recognition and Machine Learning (Information Science and Statistics)," SpringerVerlag New York, Inc., Secaucus, NJ, USA., 2006.

10. A. Alvarado et al., "LDPC Codes for Optical Channels: Is the FEC Limit a Good Predictor of Post-FEC BER?" Proc. OFC/NFOEC, 2015. 